\title{
The connection of depression and heart disease and its implications
}

\author{
HILLEL W. COHEN and JAMES DAVID
}

The link between emotion (affect) and the heart is not a new story. Folklore in a number of cultures considered the heart as the organ of emotion. "A merry heart doeth good like a medicine: but a broken spirit drieth the bones" appears in the biblical Proverbs (17:22), and Shakespeare observed “... a light heart lives long." (Loves Labor Lost). In the last fifteen years this link has become the focus of a growing literature of prospective, epidemiological studies (Carney et al., 1988; Thomas et al., 1992; Anda et al., 1993; Aromaa et al., 1994; Vogt et al., 1994; Frasure-Smith et al., 1995; Lapane et al., 1995; Wassertheil-Smoller et al., 1996; Barefoot \& Schroll, 1996; Pratt et al., 1996; Bingefors et al., 1996; Ford et al., 1998; Sesso et al., 1998; Cohen et al., 2000; Penninx et al., 2001). Almost all have reported some positive, statistically significant association of depression with cardiovascular disease (CVD), although one found the association with change in depression status rather than baseline depression (Wassertheil-Smoller et al., 1996), and another found the association with all-cause mortality but not CVD mortality (Vogt et al., 1994). One did not report any association at all (Thomas et al., 1992). This latter finding could be a result of characteristics of the sample or related to the study methods. It also could be simply an exception. Despite modest variation, the reported results are remarkably consistent, especially since the studies were conducted by different research teams, and used different measures of depression and CVD outcome. The consistency of findings is even more remarkable due to the widely varying study populations that included those with and without pre-existing heart disease; those with

Indirizzo per la corrispondenza: Professor H.W. Cohen, Albert Einstein College of Medicine, Bronx, New York (USA).

Fax: +1-718-430-3052

E-mail: hicohen@aecom.yu.edu and without hypertension; disparate occupations (a range that included medical students /doctors and lower-paid service workers); and that had varying age, gender and ethnic distributions.

As an example of the evidence linking depression with CVD, we will present data from the Worksite Hypertension Program in a study conducted by a research team at the Albert Einstein College of Medicine in New York City (Cohen et al., 2001a).

The Worksite Hypertension Program began in 1973 as a union-sponsored, nurse-managed systematic treatment program for patients with hypertension. Over the course of 25 years, more than 12000 participants received antihypertensive treatment in the program. At the time of this study, the mean follow-up time was 5 years with a range of 6 months to 25 years. Although primarily a treatment program, Worksite had a research orientation from the outset. Details of the program have been described elsewhere among the many studies of CVD outcomes that have been published from analyses of the program data. (Alderman \& Schoenbaum, 1975; Alderman et al., 1991; Cohen et al., 2001b).

In 1981, the entry medical history questionnaire was modified to include a question about history of treatment for depression. Subjects in this study were those who entered the hypertension treatment program in the years 1981-1994 and who answered the history of depression question. Of the 6,837 patients who met these criteria, 55 patients responded "uncertain" to the depression question and 1,218 reported a history of CVD at entry. These patients were excluded from the study, leaving a study cohort of 5,564. Entry data were recorded from nurse interviews, a physician's exam, an electrocardiogram, and blood serum laboratory values. Outcome events were hospitalization or death from myocardial infarction (MI), cardiac events (MI, angioplasty or revascularization), and ischemic heart disease (IHD). Hazard ratios were calculated with Cox 
proportional hazard models, adjusting for sex, age, race, history of diabetes, smoking, left ventricular hypertrophy, cholesterol, education, hypertension treatment at entry, systolic blood pressure, alcohol problem, body mass index, blood sugar and unmarried status.

Sex-adjusted rates of MI, cardiac events and IHD were significantly higher for those with history of treatment for depression. Hazard ratios of MI, cardiac events and IHD for history of treatment for depression were 2.10 (95\% Confidence Interval 1.04, 4.23), $2.44(1.33,4.48)$ and $2.11(1.23,3.62)$ respectively. There were no significant associations between depression and non-CVD hospitalizations and deaths.

What is the underlying mechanism behind the association observed here and in the other studies? Perhaps depression leads to behaviors that increase CVD risk such as smoking, high-fat diet or lack of physical activity. Depression may inhibit behaviors such as medicine adherence that would be protective for those with hypertension, hyperlipidemia, diabetes or prior CVD. In this study, however, the associations of depression with CVD were independent of smoking, blood pressure, cholesterol, blood sugar and body mass index - variables that could be considered markers for some of these CVD risk behaviors. On the other hand, it is also possible that these variables were not adequate measures of those behaviors.

A number of other potential mechanisms have been suggested, based on associations with other factors that are in turn associated with CVD. Depression can be either a determinant or a result of social isolation (Ahern et al., 1990). An association has been found between depression and heart rate variability (Carney et al., 1995a). Hopelessness, a common dimension of depression has been found to be associated with carotid thickening, suggesting that depression may be linked to an atherosclerotic process (Everson $e t$ al., 1997). Potential associations with PAI-1, homocysteine, fibrinogen and other blood markers have been suggested although the data are scant (Carney et al., 1995b; Glassman, 1998).

Another study by the same research team at Einstein, but with a different population had results that were consistent with a mechanism unrelated to depressive symptoms and behaviors (Cohen et al., 2000). Pharmacy records for a large population $(n=52,750)$ of health workers were examined. Those who had a prescription for an antidepressant during a two year accrual period $(n=2,247)$ were considered the exposed group. After 3.5 years of average follow-up, the group with antidepressant use had an adjusted hazard ratio for MI of 1.8 (95\% CI 1.1-3.1). Especially intriguing was the finding that the increased risk was observed for those taking tricyclic antidepressants (TCA) (hazard ratio $2.2,95 \% \mathrm{CI} 1.2,3.8$ ) whereas those taking selective serotonin reuptake inhibitors (SSRI) did not show a statistically significant increased risk (hazard ratio $0.8,95 \%$ CI $0.2,3.5$ ). Results were similar for all cardiovascular hospitalizations or death.

Others have also observed differences in CVD outcomes between SSRI and other antidepressants, with better outcomes associated with SSRIs (Roose et al., 1998; Sauer et al., 2001). TCAs behave as a class-I antiarrythmic agent. These agents have been known to produce adverse arrythmias in some patients and heart conduction problems are a known contraindication for TCA. It is possible that this side effect, alone or in combination with the reduced heart rate variability that has been observed with depression, could have cardiotoxic effects for some patients.

An alternate, though not mutually exclusive hypothesis, is that serotonin inhibition, the hallmark of SSRIs, might have an anti-platelet effect that can be protective (Musselman et al., 1996). Platelets can play a substantial role in the evolution of ischemic heart disease. (Nemeroff \& Musselman, 2000). Platelet aggregation and thrombosis at active plaque sites in the arterial wall contributes to atherosclerosis. Acute thrombosis that takes place in already narrowed arteries can trigger myocardial infarction or ischemic stroke. Serotonin is released by activated platelets and leads to further platelet activation. It is plausible that the altered serotonin dynamics that occur in depressed patients simultaneously alters platelet activity. The mechanism by which SSRIs are effective in the treatment of depression may also prove to be cardioprotective. Such processes might account in part for the observed depression-CVD association and for the potential benefit of SSRI relative to TCA and other antidepressant therapies with regard to cardiovascular events even when the effects on depressive symptoms do not substantially differ between the antidepressant classes.

Two recent clinical trials were undertaken to determine whether treatment for depression would reduce the associated CVD risk. In one of these, Enhancing Recovery in Coronary Heart Disease Patients (ENRICHD) 3000 patients with postMI depression were randomized to individually tailored counseling and group sessions based on cognitive-behavioral therapy or to usual care (ENRICHD Investigators, 2000). Severely depressed patients received standardized pharmacotherapy as indicated. Preliminary results were presented at the American Heart Association (AHA) Scientific Sessions in November, 2001 (Berkman \& Jaffe, 2001). As was expected, the intervention arm showed significantly greater reduction of depressive symptoms although the usual care group also showed improvement. However, no differences in CVD outcomes were observed between the groups. 
Preliminary results of another trial, Sertraline Antidepressant Heart Attack Randomized Trial (SADHART) (Shapiro et al., 1999) were also announced at the November AHA meeting. Subjects diagnosed with depression following MI or other acute coronary syndromes were randomized to sertraline, an SSRI antidepressant, or placebo. The intervention group showed significantly lower depressive symptoms than the controls but there were no differences in ejection fraction, ventricular arrhythmias or electrocardiogram intervals. There were fewer severe CVD events observed in the treated group, but with a small number of events and limited follow-up (24 weeks), this reduction did not reach statistical significance ( $\mathrm{O}^{\prime}$ Connor et al., 2001).

These findings, though preliminary, are consistent with the hypothesis that the depression-CVD association is not primarily driven by the cardiovascularly unhealthy behaviors that may be more frequent among depressed patients. If such behaviors were the main factor, one would expect that the successful treatment of depressive symptoms would have a significant impact on CVD outcomes. On the other hand, it is possible that poor health habits adopted during depressive illness do not change as quickly as depressive symptoms and the adverse CVD effects of those behaviors may take much longer to reverse than the time of the ENRICHD trial. The preliminary findings of SADHART, while negative for the intermediate measures of CVD risk, were in the direction of improved outcomes for serious CVD events, albeit not statistically significant. It is possible that the relatively short duration of follow-up so far in this trial has limited the ability to detect differences in long-term CVD outcomes. Certainly more, larger and longer trials will be needed to find out whether or how modifying depression or the antecedents of depression could improve CVD outcomes.

Until more is known, can the observed associations of depression and CVD help guide individual and public health practice?

The great majority of antidepressants are prescribed by non-psychiatrists, and concurrent with the advent of the SSRIs, which are safer than the antecedent TCAs, there has been increased prescribing by all groups. Considerations about the modification of screening and prescribing practices are relevant not only to psychiatrists, but to cardiologists, general internists, family practitioners, and to all those prescribing either cardiac or psychotropic medications. Patients with either CVD or depression deserve notice from their health care providers that the presence of one condition may put them at added risk of the other. At the time of diagnosis, CVD patients are customarily invited to reconsider smoking, serum lipid levels, obesity, and other factors; with the hope that, with one's life in the balance, there could be renewed motivation to quit smoking, lose weight, or similarly take constructive action. We assert that untreated depression should be added to this checklist, and suggest much benefit at little risk or cost would accrue if diagnosing and treating depressive disorders were added to the list of targeted behaviors. Further, if antidepressant medication is indicated for patients with CVD or CVD risk, the evidence suggesting that SSRIs are superior to TCAs must inform clinical practice.

Newly diagnosed depression patients are the mirror image of the newly diagnosed CVD patients discussed above, and could be made aware of the association of this mood disorder with CVD and counseled to seek cardiac evaluation. This could conceivably spur reluctant patients to more fully participate in depression treatment, having been informed that cardiac and mental health are intertwined. If one believes that such therapy may improve longevity and reduce the risk of a fatal or disabling heart attack or stroke, one is arguably more likely to undertake and stay with the therapy.

The medical care-seeking public has learned that unchecked smoking shortens lives. And many patients who smoke reconsider or quit when diagnosed with a cardiac condition, with the epidemiologic association now well known. Many patients would benefit if the association between CVD and depression were similarly well known to the public. It would strengthen the motivation for depressed patients to seek treatment for depressive symptoms, if the risk of CVD consequences were envisioned. The CVD-depression association lends much-needed legitimacy to patients seeking help for depression. Conversely, the physicians charged with advising cardiac patients would do well to persuade these individuals to be alert to the signs of depression and seek treatment accordingly.

As more becomes known about the CVD-depression association, more specific and definitive recommendations may be indicated. Until such time, the evidence available now is sufficiently strong to motivate providers and patients alike to more closely follow already established practice for the prevention and treatment of these two conditions that account for such a terrible burden in suffering and mortality all over the world.

\section{REFERENCES}

Ahern D., Gorkin L., Anderson J., Tierney C., Hallstrom A., Ewart C., Capone R.J., Schron E., Kornfeld D., Herd J.A., et al. (1990). 
Biobehavioral variables and mortality or cardiac arrest in the Cardiac Arrhythmia Pilot Study (CAPS). American Journal of Cardiology 66, 59-62.

Alderman M.H. \& Schoenbaum E.E. (1975). Detection and treatment of hypertension at the worksite. New England Journal of Medicine $293,65-68$.

Alderman M.H., Madhavan S., Ooi W.L., Cohen H., Sealey J.E. \& Laragh J.H. (1991). Association of the renin-sodium profile with the risk of myocardial infarction in patients with hypertension. New England Journal of Medicine 324, 1098-1104.

Anda R., Williamson D., Jones D., Macera C., Eaker E., Glassman A. \& Marks J. (1993). Depressed affect, hopelessness, and the risk of ischemic heart disease in a cohort of U.S. adults. Epidemiology 4, 285294.

Aromaa A., Raitasalo R., Reunanen A., Impivaara O., Heliovaara M., Knekt P., Lehtinen V. Joukamaa M. \& Maatela J. (1994). Depression and cardiovascular diseases. Acta Psychiatrica Scandinavica, Supplementum No. 377, 77-82.

Barefoot J.C. \& Schroll M. (1996). Symptoms of depression, acute myocardial infarction, and total mortality in a community sample. Circulation 93, 1976-1980.

Berkman L.F. \& Jaffe A.S., for the ENRICHD Investigators (2001) The effects of treating depression and low social support on clinical events after a myocardial infarction (Abstract). Circulation 2001 $104(25), 1 B$.

Bingefors K., Isacson D., Von Knorring L., Smedby B. \&Wicknertz K. (1996). Antidepressant-treated patients in ambulatory care. Mortality during a nine-year period after first treatment. British Journal of Psychiatry 169, 647-654.

Camey R.M., Rich M.W., Freedland K.E., Saini J., teVelde A., Simeone C. \& Clark K. (1988). Major depressive disorder predicts cardiac events in patients with coronary artery disease. Psychosomatic Medicine 50, 627-633.

Carney R.M., Saunders R.D., Freedland K.E., Stein P., Rich M.W. \& Jaffe A.S. (1995a). Association of depression with reduced heart rate variability in coronary artery disease. American Journal of Cardiology 76, 562-564.

Carney R.M., Rich M.W. \& Jaffe A.S. (1995b). Depression as a risk factor for cardiac events in established coronary heart disease: a review of possible mechanisms. Annals of Behavioral Medicine 17, 142-149.

Cohen H.W., Gibson G. \& Alderman M.H. (2000). Excess risk of myocardial infarction in patients treated with antidepressant medications: association with use of tricyclic agents. American Journal of Medicine $108,2-8$.

Cohen H.W., Madhavan S. \& Alderman M.N. (2001a). History of treatment for depression: risk factor for myocardial infarction in hypertensive patients. Psychosomatic Medicine 63, 203-209.

Cohen H.W., Madhavan S. \& Alderman M.H. (2001b). High and low serum potassium associated with cardiovascular events in diuretictreated patients. Journal of Hypertension 19, 1315-1323.

ENRICHD Investigators (2000). Enhancing recovery in coronary heart disease (ENRICHD): study design and methods. American Heart Journal 139 (1), 1-9.

Everson S.A., Kaplan G.A., Goldberg D.E., Salonen R. \& Salonen J.T. (1997). Hopelessness and 4-year progression of carotid atherosclerosis. Arteriosclerosis and Thrombosis 17, 1490-1495.

Ford D.E., Mead L.A., Chang P.P., Cooper-Patrick L., Wang N.Y. \& Klag M.J. (1998). Depression is a risk factor for coronary artery disease in men: the Precursors Study. Archives of Internal Medicine 158, $1422-$ 1426.

Frasure-Smith N., Lesperance F. \& Talajic M. (1995). Depression and 18-month prognosis after myocardial infarction. Circulation 91(4), 999-1005.

Glassman A.H. (1998). Depression andd the course of coronary artery disease. American Journal of Psychiatry 155, 4-11.

Lapane K.L., Zierler S., Lasater T.M., Barbour M.M., Carleton R. \& Hume A.L. (1995). Is the use of psychotropic drugs associated with increased risk of ischemic heart disease? Epidemiology 6(4), 376-381.

Musselman D.L., Tomer A., Manatunga A.K., Knight B.T., Porter M.R., Kasey S., Marzic U., Harker L.A. \& Nemeroff C.G. (1996). Exaggerated platelet reactivity in major depression. American Journal of Psychiatry $153,1313-1317$.

Nemeroff C.B. \& Musselman D.L. (2000). Are platelets the link between depression and ischemic heart disease? American Heart Journal 140, Suppl. 4, 57-62.

O'Connor C.M., Glassman A.H., Harrison W.M., for the SADHART Investigators (2001). A randomized double-blind placebo controlled trial of the selective serotonin reuptake inhibitor (SSRI) sertraline for major depression after acute coronary syndromes (ACS). In The SADHART Trial. American Heart Association Scientific Sessions 2001, Nov. 11, 2001 Abstract 102513, Anaheim, California.

Penninx B.W., Beekman A.T., Honig A., Deeg D.J., Schoevers R.A., van Eijk J.T. \& van Tilbur. Depression and cardiac mortality: results from a community-based longitudinal study. Archives of General Psychiatry 58(3), 229-230.

Pratt L.A., Ford D.E., Crum R.M., Armenian H.K., Gallo J.J. \& Eaton W.W. (1996). Depression, psychotropic medication, and risk of myocardial infarction: prospective data from the Baltimore ECA follow-up. Circulation 94, 3123-3129.

Roose S.P., Laghrissi-Thode F., Kennedy J.S. et al., (1998). Comparison of paroxetine and nortriptyline in depressed patients with ischemic heart disease. Journal of the American Medical Association 279, 287 291.

Sauer W.H., Berlin J.A. \& Kimmel S.E. (2001). Selective serotonin reuptake inhibitors and myocardial infarction. Circulation 104(16), 1894-1898.

Sesso H.D., Kawachi I., Vokonan P.S. \& Sparrow D. (1998). Depression and the risk of coronary heart disease in the Normative Aging Study. American Journal of Cardiology 82(7), 851-856.

Shapiro P.A., Lesperance F., Frasure-Smith N., O'Connor C.M., Baker B., Jiang W., Dorian P., Harrison W. \& Glassman A.H. (1999). An open-label preliminary trial of sertraline for treatment of major depression after acute myocardial infarction (the SADHART Trial). Sertraline Anti-Depression Heart Attack Trial. American Heart Journal 137, 1100-1106.

Thomas C., Kelman H.R., Kennedy G.J., Ahn C. \& Yang C. (1992). Depressive symptoms and mortality in elderly persons. Journal of Gerontology 47(2), S80-S87.

Vogt T., Pope C., Mullooly J. \& Hollis J. (1994). Mental health status as a predictor of morbidity and mortality: a 15-year follow-up of members of a health maintenance organization. American Journal of Public Health 84, 227-231.

Wassertheil-Smoller S., Applegate W.B., Berge K., Chang C.J., Davis B.R., Grimm R. Jr, Kostis J., Pressel S. \& Schron E. (1996). Change in depression as a precursor of cardiovascular events. Archives of Internal Medicine 156,553-561. 